\title{
Opción de responsabilidad limitada y opción de abandonar: un análisis a través de opciones americanas perpétuas
}

\author{
Neus Orgaz \\ Universidad Autónoma de Barcelona \\ Facultad de Ciencias Económicas
}

\begin{abstract}
RESUMEN
Este documento analiza la opción de responsabilidad limitada de los accionistas de una sociedad anónima endeudada y la opción de abandonar la actividad empresarial a través de la opción de venta americana perpetúa. Las propiedades del análisis de la quiebra empresarial mediante las opciones perpetuas se comparan con el análisis en un horizonte finito. Se introduce los costes fijos en la opción de abandono empresarial. La integración de la opción de responsabilidad y la opción de abandono permite analizar los diferentes escenarios de crisis empresarial desde el punto de vista del valor.

Clasificación JEL: G330

Palabras claves: quiebra empresarial, opción de abandonar, opciones americanas.
\end{abstract}

\begin{abstract}
This work studies the default option and the abandonment option by means of perpetual American puts, which are valued according to Merton's formula. The effect of operating fixed costs is introduced in the abandonment option. The properties of default analysed through perpetual puts are compared with the finite horizon case.
\end{abstract}


The joint consideration of default and abandonment option enables to analyse the different scenarios of the firm's crisis from the value point of view. The distance from default that stems from the perpetual default option is calculated and, next, related to the accounting leverage ratio and the asset's market/book ratio.

JEL Classification: G330

Key wordds: quiebra empresarial, opción de abandonar, opciones americanas.

\section{INTRODUCCIÓN}

El objetivo fundamental de este artículo es analizar los diferentes escenarios de crisis en la capacidad de la empresa para generar valor, utilizando la teoría de valoración de opciones. Las crisis empresariales se enfocan en este trabajo desde el punto de vista del valor y se concretan en la quiebra, por una parte, y en el cese de actividades, por otra.

Los modelos desarrollados en este trabajo tratan de profundizar en el derecho de responsabilidad limitada de los accionistas, que se analiza a partir de los conocimientos que proporciona la teoría de opciones. La responsabilidad limitada de los accionistas aparece asociada a la declaración de quiebra. La suspensión de pagos debe verse como un intento de evitar la quiebra y pertenece, por tanto, en el enfoque de este trabajo al mismo tipo de crisis de valor que la quiebra.

Las crisis empresariales se concretan, pues, en la quiebra y el cese de actividades. Como se pone de manifiesto en el trabajo, ambas situaciones no deben asimilarse. Puede darse una situación de quiebra sin cese de actividades, porque los acreedores, como consecuencia de la quiebra, reciben de los accionistas una empresa que, aún no habiendo sido capaz de satisfacerles las deudas, es todavía viable ya que ni el valor de venta en el mercado de los elementos del activo ni el volumen de los costes fijos justifican el cese. El cese de actividades puede, por su parte, no ir asociado a la quiebra, como ocurre en el caso en que el valor de liquidación del activo es superior al valor de las deudas. La quiebra no debe entenderse aquí como una acción judicial sino ante todo como un cambio de propiedad que ha implicado o no trámites judiciales.

En el análisis de la quiebra, Black y Scholes (1973) abren un camino mediante su propuesta para valorar las acciones de una empresa endeudada como una opción de compra europea sobre su activo con un precio de ejercicio igual al pago prometido a los acreedores, en un único período. A partir de este resultado y utilizando la paridad put-call, es posible valorar la opción de responsabilidad limitada de las sociedades 
anónimas como una opción de venta europea y, asimismo, calcular la rentabilidad exigida para la financiación ajena.

La principal ventaja del planteamiento de Black y Scholes (1973) radica en la aplicación de la teoría de opciones a la valoración de las acciones de las empresas endeudadas, a la valoración de la limitación de responsabilidad de los accionistas de una sociedad anónima y a la determinación de la rentabilidad exigida. Merece destacarse que la limitación de responsabilidad no ha podido valorarse por métodos alternativos a la teoría de opciones. Sin embargo, el modelo de Black y Scholes (1973) presenta algunas limitaciones significativas: horizonte a un período, tipo de interés constante y volatilidad constante.

El horizonte de la empresa es, en principio, ilimitado, concepto que está ampliamente reconocido en los modelos de valoración de acciones. Es frecuente que se mantenga un determinado grado de endeudamiento a lo largo de su vida, por lo que se puede considerar el endeudamiento de la empresa de horizonte perpetuo. Como consecuencia, los accionistas pueden declarar la quiebra en cualquier momento, es decir, ejercer la opción de responsabilidad limitada y dejar la empresa en manos de los acreedores.

Por tanto, en este trabajo se analiza:

- las consecuencias de concebir la opción de responsabilidad limitada como una opción de venta americana de horizonte perpetuo,

- la decisión de continuar o cesar la actividad de la empresa a partir de la opción de abandonar, que conduce a comparar el valor de la empresa en funcionamiento con el valor de venta del activo en el mercado, es decir, el valor que se obtiene vendiendo el activo y liquidando, pues, la empresa,

- la opción de abandonar como una opción de venta americana, cuyo precio de ejercicio incorpora no solo el valor de liquidación de la empresa, sino también el efecto de los costes fijos, y

- la comparación de ambas opciones, lo cual nos permite sistematizar los diferentes escenarios de la crisis de la empresa que se origina en su valor.

La estructura de este trabajo es la siguiente: en la primera sección se realiza una revisión literaria. En la segunda sección se analiza la opción de responsabilidad limitada en un horizonte perpetuo. En la tercera sección se analiza la decisión de abandonar la actividad de la empresa y los diferentes escenarios de crisis de valor de la empresa. En la cuarta sección se calcula la distancia de la quiebra, y la última sección, las conclusiones. 


\section{REVISIÓN LITERARIA}

Este trabajo de investigación se enmarca dentro de la teoría de opciones y basa su análisis para la construcción de los modelos que en él se desarrollan en tres pilares de este campo: el modelo de valoración de opciones de Black y Scholes (1973); los modelos de Merton para valorar opciones de venta americanas en un horizonte perpetuo (1973) y la deuda empresarial $(1974,1977)$; y las opciones de barrera. Black y Scholes (1973), juntamente con Merton (1973) fueron los pioneros de la moderna teoría de valoración de opciones.

El modelo de valoración de opciones que Black y Scholes dieron a conocer 1973, ha facilitado y ayudado al crecimiento de los mercados financieros de derivados, ya que muchos modelos de valoración de opciones se basan directa o indirectamente en él. Sin embargo, como señalan Brealey y Myers (2006, capítulo 21), el concepto de opción no sólo da lugar a los correspondientes títulos negociados en los mercados financieros de derivados sino que constituye, además, un instrumento extremadamente útil para analizar y valorar los proyectos de inversión y la política financiera de la empresa.

Desde que Black y Scholes publicaron su artículo pionero en 1973, han surgido numerosos trabajos que han relajado las hipótesis y han extendido la fórmula de Black y Scholes.

Merton en 1973 desarrolla, entre otros, un modelo que permite valorar opciones de venta americanas en un horizonte perpetuo. En este documento, este modelo, se adapta para valorar la opción de responsabilidad limitada en un horizonte perpetuo. Merton formaliza la valoración de la deuda por teoría de opciones en sus artículos de 1974 y 1977.

El centro conceptual de este trabajo es la opción de responsabilidad limitada de los accionistas. Esta opción establece un nexo entre la rentabilidad exigida por la financiación ajena, el valor de las acciones y el riesgo de crédito. Varios son los autores que, desde enfoques distintos a los abordados en este trabajo, han estudiado diversos aspectos que atañen tanto al valor de las acciones y como al análisis la deuda en sus múltiples facetas. Obviamente, los pioneros en el análisis moderno de la relación entre el valor de las acciones y el valor de la deuda son Modigliani y Miller (1958).

Una de las cuestiones importantes en el modelo que se elabora en este trabajo es, como ya se ha mencionado, el horizonte temporal de la vida de la empresa. Generalmente, los títulos de la deuda tienen un vencimiento especificado, pero la renovación constante que las empresas realizan de la deuda permite asumir la hipótesis de deu- 
da perpetua. Así, Modigliani y Miller (1958) en sus argumentos asumen una deuda con vencimiento infinito. A su vez, Merton (1974) y Black y Cox (1976) suponen también una deuda con vencimiento infinito en un modelo dinámico.

Existen, sin embargo, otras aportaciones relevantes al estudio del valor de las acciones y de la deuda. Leland (1994) examina el valor de la deuda y la estructura de capital. Obtiene resultados para valorar la deuda a largo plazo, la rentabilidad y su estructura óptima de capital, cuando la rentabilidad de la empresa sigue un proceso con volatilidad constante.

Brennan y Schwartz (1978) realizan un examen cuantitativo del nivel óptimo del endeudamiento. Utilizan técnicas numéricas para determinar el nivel óptimo de la estructura de capital con volatilidad constante.

Este trabajo se puede conectar con las opciones reales: Dixit y Pyndyck (1994) valoran la decisión de realizar o no proyectos de inversión en un escenario incierto, siguiendo la teoría de opciones y teniendo en cuenta que el valor de mercado de un proyecto al igual que el valor de mercado del activo de la empresa siguen un proceso estocástico.

Mella-Barral y Perraudin (1997), en su artículo construyen un modelo de valoración de activos e incorporan estrategias de deuda. Trabajan en tiempo continuo y obtienen expresiones para valorar la deuda y las acciones, cuando la deuda es perpetua. En sus análisis incorporan los costes directos de quiebra y los costes de agencia.

Longstaff y Schwartz (1995) desarrollan una investigación para valorar el riesgo de crédito de una empresa, que incorpora la quiebra y el tipo de interés con riesgo. El valor residual de la empresa se incorpora en la situación de quiebra.

Duffee (1999) elabora un modelo para estimar la probabilidad de quiebra de una empresa. Los parámetros del proceso están estimados para 161 empresas. En cada instante, hay alguna probabilidad de que la empresa quiebre por no poder hacer frente a sus obligaciones. El proceso estocástico determina el precio del riesgo de crédito.

Uno de los inconvenientes de estas investigaciones surge por el hecho de considerar que la empresa declara la quiebra cuando ha agotado todo su activo. Esto no se corresponde exactamente con la realidad, en la medida en que muchas empresas quiebran antes de haber agotado la totalidad de los activos. Atendiendo precisamente a este aspecto, Black y Cox (1976) relajan este supuesto y consideran que la quiebra ocurre cuando el valor de los activos alcanza un umbral bajo.

Jones, Mason y Rosenfield (1984) tienen como objetivo verificar el poder de predicción de la teoría clásica de Black y Scholes (1973) y Merton (1974, 1977). Por tanto, su punto de partida y el de este trabajo comparten modelos comunes; en cambio, tanto la metodología empleada como los objetivos de ambos trabajos son distintos. 
Eliasson, Gao y Song (2002) analizan las quiebras voluntarias de la empresa, y valoran esta decisión voluntaria de liquidación de la empresa cómo una opción de venta americana.

\section{LA OPCIÓN DE RESPONSABILIDAD LIMITADA EN UN HORIZONTE PERPETUO}

Proponemos en primer lugar el modelo básico que se basa en que el horizonte de la empresa es, en principio, ilimitado, y por tanto consideramos que el endeudamiento de la empresa es de horizonte perpetuo. El derecho de responsabilidad limitada de los accionistas se asimila a una opción de venta americana de horizonte perpetuo sobre el activo de la empresa con un precio de ejercicio igual al pago prometido a los acreedores. Los accionistas son propietarios del activo de la empresa, pagan los intereses de la deuda, y adquieren la opción de responsabilidad limitada emitida por los acreedores.

La opción de responsabilidad limitada, $\mathrm{F}_{\infty}$; puede valorarse aplicando la fórmula de la opción de venta americana perpetúa deducida por Merton (1973 y 1990 págs. 298-300), siendo:
$\mathrm{A}=$ valor del activo de la empresa (activo subyacente)
$\mathrm{D}=$ valor de la deuda nominal (precio de ejercicio)
$\mathrm{r}=$ tipo de interés libre de riesgo
$\sigma=$ desviación típica de la rentabilidad del activo (A).

La expresión del valor de la opción de responsabilidad limitada ${ }^{1}$ como una opción de venta americana perpetua es la siguiente:

$$
F_{\infty}=\left[\frac{D}{1+\gamma}\right] \cdot\left[\frac{(1+\gamma) \cdot A}{\gamma \cdot D}\right]^{-\gamma}
$$

${ }^{1}$ En base de la opción de responsabilidad limitada perpetua, la rentabilidad exigida de la deuda $(k)$ puede ser calculada de la siguiente manera:

$$
D_{0} \cdot k=r_{0} \cdot\left(D_{0}+F_{\infty}\right)
$$


Siguiendo la notación introducida por Merton,

$$
\gamma=\frac{2 \cdot r}{\sigma^{2}}
$$

De acuerdo con esta proposición, el valor de las acciones es igual al valor del activo con la opción de responsabilidad limitada menos el valor de la deuda. Puede justificarse que la suma del valor del activo con la opción de responsabilidad limitada equivale a una opción de compra americana perpetua sobre el activo con una barrera inferior de salida igual al valor máximo del activo en el que se declara la quiebra y una compensación (rebate) igual al valor de la deuda, como indica Crosbie y Bohn (2002). Por tanto, las acciones pueden concebirse como la diferencia entre el valor de la opción de compra perpetua y de barrera y el endeudamiento personal de los accionistas en condiciones de responsabilidad ilimitada. Lógicamente, los propietarios de esta opción son los accionistas y los emisores son los acreedores. Cuando el activo alcanza el valor de la barrera y se produce la situación de quiebra, la opción desaparece. Por su parte, la compensación permite a los accionistas eliminar su endeudamiento personal, es decir, los accionistas pierden sus derechos sobre el activo y reciben una cantidad que les permite saldar sus deudas con los acreedores.

\subsection{El ejercicio de la opción de responsabilidad limitada en un horizonte perpetuo: la declaración de quiebra}

Merton (1973) en su trabajo determina el valor máximo del activo subyacente que justifica un ejercicio anticipado de la opción de venta americana de horizonte perpetuo. Aplicando este resultado a la opción de responsabilidad limitada, el valor máximo del activo de la empresa para que se declare en quiebra es el siguiente:

$$
A^{*}=\frac{\gamma \cdot D}{1+\gamma}
$$

Sustituyendo $A^{*}$ en $F_{\infty}$ obtenemos el valor de la opción de responsabilidad limitada en el momento de la quiebra: 


$$
F_{\infty} *=\frac{D}{1+\gamma}
$$

Este resultado es coherente con el hecho que en el momento de quiebra el valor de las acciones es cero. Como se ha mencionado anteriormente, el valor de las acciones es la suma del valor del activo más la opción de responsabilidad limitada menos el valor de la deuda. Si sumamos el valor del activo en caso de quiebra, ecuación (3), con el valor de la opción de responsabilidad limitada en la misma circunstancia, ecuación (4), se obtiene el valor de la deuda, por lo que se deduce que el valor de las acciones es cero.

Cuando se produce una situación de quiebra, donde los accionistas han ejercitado su opción de responsabilidad limitada, los acreedores, por su posición de vendedores de la opción de responsabilidad limitada, están obligados a adquirir el activo a cambio de la deuda. Como consecuencia, los acreedores experimentan la siguiente pérdida:

$$
\frac{\gamma \cdot D}{1+\gamma}-D=-\frac{D}{1+\gamma}
$$

Es decir, la pérdida que experimentan los acreedores en caso de quiebra es igual al valor de la opción de responsabilidad limitada cuando se ejercita anticipadamente.

En el caso de las opciones europeas los accionistas declaran la quiebra si al final del período el valor del activo de la empresa (activo subyacente) es inferior o igual al pago prometido a los acreedores (valor de la deuda):

$$
A_{T}<D
$$

Mientras que en el modelo perpetuo los accionistas declaran la quiebra en aquel momento, coincidente o no con el final del período, en que el activo se sitúa en un valor inferior o igual, expresado en la ecuación (3), 


\subsection{Vencimiento finito de la deuda y quiebra}

Sea una empresa que se ha endeudado a un horizonte finito de modo que la totalidad de su deuda vence a final del período en curso, $T$. El valor de la opción de responsabilidad limitada europea en el momento del vencimiento es:

$$
\operatorname{Max}\left[0, D-A_{T}\right]
$$

Supongamos que al llegar el vencimiento, momento $T$, de la deuda tenemos:

$$
\frac{\gamma \cdot D}{1+\gamma}<A_{T}<D
$$

Esto quiere decir, que el valor del activo de la empresa es inferior al valor de la deuda, pero es superior al valor del activo que justifica la quiebra en el modelo perpetuo. Resulta entonces que la quiebra está justificada desde el punto de vista de la opción de responsabilidad limitada europea de vencimiento finito. Sin embargo, no lo está desde el punto de vista de la opción de responsabilidad limitada perpetua. Los accionistas puede, ciertamente, optar por la declaración de quiebra, pero el hecho de que esta empresa es financieramente viable desde el punto de vista de la opción de responsabilidad limitada perpetua abre otra posibilidad que consiste en la siguiente estrategia como alternativa a la quiebra:

a) Los accionistas adquieren la opción de responsabilidad limitada perpetua a los acreedores.

b) En pago de la opción perpetua que acaban de adquirir les entregan la opción de horizonte finito que vence en este momento, cuyo valor es la diferencia entre las deudas y el activo, $D-A_{T}$.

c) El valor de la opción perpetua es superior al valor de la opción finita, los accionistas pagan en efectivo la diferencia: $\mathrm{F}_{\infty}-\left(D-A_{T}\right)$.

De esta manera, los accionistas continúan como propietarios de las acciones de la empresa, tras pagar la opción de responsabilidad limitada perpetua adquirida en 
este proceso. Este resultado remarca que la quiebra es solo viable en el caso que el valor del activo de la empresa sea igual o inferior a (3), el valor máximo que justifica el ejercicio anticipado de la opción de responsabilidad limitada perpetua.

\section{LA DECISIÓN DE ABANDONAR}

En el anterior punto se ha analizado la opción de responsabilidad limitada en un horizonte perpetuo sin tener en cuenta la opción que tiene la empresa de decidir el abandono de la misma, ni los costes fijos. En este apartado, introducimos dos conceptos significativos: la opción de abandonar y los costes fijos. Dixit y Pindyck (1994) en su proceso de valoración de proyectos de inversión también realizan el mismo proceso: primero realizan un análisis sin costes, y posteriormente se introducen.

La opción de abandonar se introduce de la siguiente manera: se toma el valor de venta que tiene el activo de la empresa en el mercado, los accionistas lo comparan con el valor de las deudas y deciden si es más rentable seguir con la empresa o vender los elementos del activo en el mercado. Los costes fijos pueden asimilarse a una deuda perpetua, cuyo interés es precisamente el importe de los costes fijos y sobre la que los accionistas gozan de la opción de responsabilidad limitada. La consideración de los costes fijos nos permite analizar las causas del cese de las actividades de la empresa, por una parte, y de la quiebra, por otra.

En la construcción de este nuevo modelo y la valoración de las diferentes opciones se utiliza la fórmula deducida por Merton (1973) de la opción de venta americana perpetua.

\subsection{Introducción de los costes fijos}

Introducimos los costes fijos en el análisis del valor de la opción de responsabilidad limitada. Los pagos constantes a que dan lugar los costes fijos $(F C)$ se asimilan a intereses sobre una deuda perpetua que denominamos deuda estructural $(S D)$. Los costes fijos obligan a realizar un pago permanente que los accionistas solo puede dejar de satisfacer cesando la actividad de la empresa, es decir, abandonando la empresa. Por tanto, los costes fijos pueden asimilarse a una deuda perpetua cuyo interés anual es precisamente el importe de los costes fijos y sobre la que los accionistas gozan de la opción de responsabilidad limitada.

Para definir esta opción debemos definir previamente el valor del activo antes de 
la incidencia de los costes fijos, es decir, el valor actual de la renta que resulta de la diferencia entre ingresos y costes variables. Denominamos a este activo, activo de coste variable $(A V C)$. Su valor, en el caso en que los valores esperados de los ingresos $(I N)$ y los costes variables $(V C)$ sean constantes en un horizonte perpetuo, es:

$$
A V C=\frac{I N-V C}{\bar{R}_{A}}
$$

donde es la rentabilidad exigida del activo $^{2}$.

El valor de la deuda estructural es:

$$
S D=\frac{F C}{r}
$$

El valor del activo después de la incidencia de los costes fijos se obtiene restando el valor de la deuda estructural del valor del activo de coste variable:

$$
A=A V C-S D
$$

El derecho de responsabilidad limitada de la deuda estructural que poseen los accionistas puede ser concebida como una opción de venta americana de horizonte perpetuo sobre el activo (de los costes variables)y con un precio de ejercicio igual al valor nominal de la deuda estructural. Por tanto, aplicando la fórmula deducida por Merton (1073, 1990), el valor de la opción de responsabilidad limitada sobre la deuda estructural es:

$$
F_{S D}=\frac{S D}{1+\gamma} \cdot\left[\frac{A V C \cdot(1+\gamma)}{\gamma \cdot S D}\right]^{-\gamma}
$$

${ }^{2}$ La aplicación práctica de este resultado requiere estimar la rentabilidad exigida del activo de la empresa antes de la incidencia de los costes fijos. 


\subsection{El cese de la actividad de la empresa: costes fijos y opción de abandonar}

La opción de responsabilidad limitada con la deuda estructural es, de hecho, una opción de abandonar que permite a los accionistas ser «liberados» de la deuda estructural cesando la actividad de la empresa. Sin embargo, el cese de la actividad empresarial también significa vender por separado los activos, entonces, significa un proceso de liquidación.

Consideramos en primer lugar, el caso de una empresa no endeudada. La posición de los accionistas es la siguiente: son propietarios del activo de coste variable, se comprometen a pagar los costes fijos, es decir, asumen la deuda estructural; gozan del derecho de la responsabilidad limitada sobre la deuda estructural, y son propietarios de una opción de abandonar.

El derecho de responsabilidad limitada sobre la deuda estructural puede integrarse en la opción de abandonar. Basta, para ello, definir la opción de abandonar como una opción de venta perpetua sobre el activo de coste variable, cuyo precio de ejercicio consiste en la suma del valor de liquidación $(L V)$ y la deuda estructural. El valor de la opción de abandonar ( $A O)$ se obtiene sustituyendo en (12) el nuevo precio de ejercicio:

$$
A O=\frac{S D+L V}{1+\gamma} \cdot\left(\frac{A V C \cdot(1+\gamma)}{\gamma \cdot(S D+L V)}\right)^{-\gamma}
$$

El máximo valor del activo que justifica el ejercicio de la opción de abandonar y, por tanto, el cese de las actividades de la empresa es:

$$
A V C^{*}=\frac{\gamma}{1+\gamma} \cdot(S D+L V)
$$




\subsection{Deuda Financiera, costes fijos y opción de abandonar}

Consideramos el caso de una empresa endeudada. La modelización mediante opciones de los efectos sobre el valor de la empresa de la deuda financiera, los costes fijos y el valor de liquidación permite analizar las causas y las consecuencias para accionistas y acreedores del cese de actividades y la quiebra de la empresa. Para llevar a cabo este objetivo, analizamos los diferentes casos que resultan de comparar el valor de liquidación, la deuda financiera y la deuda estructural.

Cese y quiebra no siempre aparecen asociados. En el contexto que resulta de la introducción de los costes fijos, tres casos son posibles: quiebra sin cese, cese sin quiebra, y cese y quiebra simultáneos.

\subsubsection{Quiebra y cese de la actividad}

Consideramos una empresa endeudada con costes fijos y valor de liquidación. Dos umbrales determina la crisis de la empresa: el valor máximo del activo que justifica el ejercicio de la opción de abandonar y el valor máximo del activo que justifica el valor de la opción de responsabilidad limitada.

Sabemos por la ecuación (14) el límite que determina el abandono. El límite que determina la quiebra requiere un análisis de la opción de responsabilidad limitada en este escenario. El activo subyacente de la opción de responsabilidad limitada con opción de abandonar y los costes fijos (AS) consiste en la suma del activo de la empresa más la opción de abandonar que incorpora el efecto de los costes fijos (13).

La opción de responsabilidad limitada con opción de abandonar cuando se consideran los costes fijos consiste en una opción de venta cuyo activo subyacente, $A S$, viene dado por la suma del activo con la opción de abandonar que incorpora el efecto de los costes fijos, $A O$ :

$$
A S=A+A O
$$

El precio de ejercicio de esta opción continúa siendo el valor de la deuda y, en consecuencia, el valor máximo del activo que provoca la quiebra es el siguiente: 


$$
A S^{* \prime}=\frac{\gamma^{\prime} \cdot D}{1+\gamma^{\prime}}
$$

donde:

$$
\gamma^{\prime}=\frac{2 \cdot r}{\sigma^{\prime}}
$$

Siendo la desviación típica del activo:

$$
\sigma^{\prime}=\frac{A \cdot\left(1-\left(\frac{(A+S D) \cdot(1+\gamma)}{(A+S D) \cdot \gamma}\right)^{-1-\gamma}\right)}{A+\frac{(S D+L V) \cdot\left(\frac{(A+S D) \cdot(1+\gamma)}{(S D+L V) \cdot \gamma}\right)^{-\gamma}}{1+\gamma} \cdot \sigma}
$$

Según se justifica en el apéndice.

Por tanto, la empresa declarará la quiebra cuando el valor del activo más el valor de la opción de abandono sea inferior a A* (ecuación 16):

$$
A+A O \leq \frac{\gamma^{\prime} \cdot D}{1+\gamma^{\prime}}
$$

Desde la ecuación (11), podemos escribir por tanto la condición de quiebra:

$$
A V C \leq \frac{\gamma^{\prime} \cdot D}{1+\gamma^{\prime}}+S D-A O
$$

Tomando la condición de abandono de la actividad empresarial (14) y la condición de quiebra (20), la quiebra domina al cese empresarial si se cumple la siguiente condición: 


$$
\frac{\gamma^{\prime}}{1+\gamma^{\prime}} \cdot D+S D-A O>\frac{\gamma}{1+\gamma} \cdot(S D+L V)
$$

Es decir, si el valor del activo que justifica la quiebra es superior al valor del activo que justifica el abandono de la actividad empresarial. De esta manera, se llega al primer escenario: quiebra sin cese. La declaración de quiebra sin cese de las actividades de la empresa se produce cuando los accionistas deciden ejercer su opción de responsabilidad limitada para un valor del activo que resulta superior a aquél que justifica el cese. Los accionistas ejercitan la opción de responsabilidad limitada declarando la quiebra. Los acreedores reciben el activo junto con la opción de abandonar y los accionistas quedan liberados del pago de la deuda.

Si se cumple la siguiente condición:

$$
\frac{\gamma^{\prime}}{1+\gamma^{\prime}} \cdot D+S D-A O<\frac{\gamma}{1+\gamma} \cdot(S D+L V)
$$

Y además, el valor de liquidación es superior a la deuda financiera, se da el caso de que el valor del activo de la empresa se sitúa en aquella cifra que justifica el ejercicio de la opción de abandonar, los accionistas ejercen esta opción y con la cantidad obtenida al realizar el valor de liquidación proceden al pago de la deuda y a distribuirse el resto. Es el caso del segundo escenario: cese sin quiebra.

Y por último, si el valor de liquidación es inferior al valor de la deuda, la decisión de abandonar implica al mismo tiempo la decisión de quiebra. Es el escenario de cese y quiebra simultáneos. En este caso, el valor de las acciones es cero. La opción de responsabilidad limitada de los accionistas les otorga el derecho a recibir una cifra igual a la diferencia entre el valor nominal de la deuda y el valor de liquidación del activo, cuando el activo alcanza el valor que da lugar al ejercicio de la opción de abandonar.

Los tres posibles casos de crisis empresariales se resumen en la tabla 1. 
Tabla 1. Casos de crisis empresariales

\begin{tabular}{|c|c|c|}
\hline$\frac{\gamma^{\prime}}{1+\gamma^{\prime}} \cdot D+S D-A O>\frac{\gamma}{1+\gamma} \cdot(S D+L V)$ & $\frac{\gamma^{\prime}}{1+\gamma^{\prime}} \cdot D+S D-A O<\frac{\gamma}{1+\gamma} \cdot(S D+L V)$ \\
\hline Caso1 & Caso 2 & \multicolumn{2}{|c|}{ Caso 3 } \\
\hline \multirow{2}{*}{$\begin{array}{c}\text { Quiebra sin cese } \\
\text { La quiebra domina al cese empresarial. }\end{array}$} & $\begin{array}{c}\text { El cese empresarial } \\
\text { domina a la quiebra. }\end{array}$ & $\begin{array}{c}\text { La quiebra y el aban- } \\
\text { dono se producen en } \\
\text { el mismo tiempo. }\end{array}$ \\
\cline { 2 - 3 } & &
\end{tabular}

\subsection{El valor de la opción de responsabilidad limitada con opción de abandonar cuando se consideran los costes fijos}

En este apartado se expresa el valor de la opción de responsabilidad limitada con opción de abandonar cuando se consideran los costes fijos. Al igual que Dixit y Pindyck (1994), aplicamos el lema de Ito para determinar el proceso estocástico del activo subyacente de la opción.

\subsubsection{Quiebra sin cese}

Cuando los accionistas deciden ejercer su opción de responsabilidad limitada para un valor del activo que resulta superior a aquél que justifica el cese. El valor de la opción de responsabilidad limitada cuando la condición de quiebra domina a la condición de abandonar la empresa es:

$$
F_{O A}=\frac{D}{1+\gamma^{\prime}} \cdot\left[\frac{\left(1+\gamma^{\prime}\right) \cdot\left[A+\frac{(S D+L V)}{1+\gamma} \cdot\left(\frac{(1+\gamma) \cdot(A+S D))}{\gamma \cdot L V}\right)^{-\gamma}\right]}{\gamma^{\prime} \cdot(F C+L V)}\right]^{-\gamma^{\prime}}
$$


Como se deduce en el apéndice.

\subsubsection{Cese y quiebra simultáneos}

En este caso el valor del activo en el momento de quiebra es superior al valor máximo que justifica el ejercicio de la opción de responsabilidad limitada y, al mismo tiempo, es inferior al valor máximo que justifica la decisión de abandonar la actividad de la empresa.

Se ha constatado que, en caso de cese y quiebra simultáneos, la opción de responsabilidad limitada de los accionistas les otorga el derecho a recibir una cifra igual a la diferencia entre el valor nominal de la deuda y el valor de liquidación del activo, cuando el activo alcanza el valor que da lugar al ejercicio de la opción de abandonar, es decir, cuando el valor del activo se sitúa en la cifra:

$$
\frac{\gamma}{1+\gamma} \cdot(S D+L V)
$$

que da lugar a la inviabilidad de la empresa.

En este caso, la opción de responsabilidad limitada puede asimilarse a una opción de venta perpetua europea, down-and-out, de barrera inferior de salida con compensación, emitida por los acreedores y siendo propietarios los accionistas. El valor de la barrera es el siguiente:

$$
\frac{\gamma}{1+\gamma} \cdot(S D+L V)
$$

Y el valor de la compensación es el siguiente:

$$
D-L V
$$

El activo subyacente de esta opción es el activo de coste variable de la empresa. Al tratarse de una opción europea perpetua nunca se ejercitará, por lo que el precio de ejercicio es irrelevante. En caso de abandono y quiebra simultáneos, indica que la barrera es cruzada, los acreedores pagan a los accionistas una cantidad $D-L V$, la compensación de la opción. Los accionistas pagan el principal de la deuda a los acreedores como consecuencia de su decisión de abandonar la actividad de la empresa. El flujo neto de las dos operaciones consiste en que los accionistas pagan a los acreedores el valor de liquidación. 
Siguiendo a Rich (1994, pág. 293) el valor de la opción de responsabilidad limitada en el caso de cese y quiebra simultáneos es el siguiente:

$$
F_{B}=(D-L V) \cdot \frac{A V C}{\frac{\gamma}{1+\gamma} \cdot(S D+L V)}
$$

\section{Distancia de la Quiebra}

El modelo presentado en este documento, se puede asociar a los enfoques actuales de cálculo del riesgo de crédito, y en concreto en el enfoque estructural que se basa en los precios de mercado de las acciones (Peña 2002, pág. 144).

Este modelo perpetuo sigue la idea básica del modelo de Merton (1974) que considera las acciones de la empresa como una opción de compra europea sobre su activo y en base a esto calcula la probabilidad de quiebra neutral al riesgo. Esta probabilidad se puede considerar como la frecuencia esperada de quiebra condicionada al valor actual de la empresa, su apalancamiento, volatilidad, estructura de la deuda y tipo libre de riesgo.

El modelo KMV (Saunders 1999 pág. 27) define la distancia hacia la quiebra como:

$$
d=\frac{A-B}{\sigma_{A}}
$$

siendo $B$ el punto donde se ejerce la opción y se declara la quiebra y $\sigma_{\mathrm{A}}$ la desviación típica del activo de la empresa. Sustituyendo $B$ por su valor en el modelo perpetuo, se obtiene:

$$
d=\frac{1}{\sigma_{A}} \cdot\left(A-\frac{\gamma \cdot D}{1+\gamma}\right)
$$


Y, después de realizar algunas operaciones ${ }^{3}$ se obtiene:

$$
d=\frac{1}{\sigma} \cdot\left(1-\frac{\gamma}{1+\gamma} \cdot \frac{1}{b}\right)
$$

donde indica el ratio contable de endeudamiento ( deuda/valor contable del activo) y $b$ indica el ratio entre el valor de mercado y contable del activo ( valor de mercado del activo/ valor contable del activo).

Esto, nos permite definir el coeficiente de quiebra como el ratio entre el valor actual de mercado del activo y el valor máximo de mercado del activo que justifica la quiebra:

$$
f=\frac{A}{\frac{\gamma}{1+\gamma} \cdot D}
$$

Es decir:

$$
f=\frac{1+\gamma}{\gamma} \cdot \frac{b}{\mathrm{I}}
$$

Y después de hacer algunas operaciones, obtenemos el cálculo de la distancia hacia la quiebra:

$$
d=\frac{1}{\sigma} \cdot\left(1-\frac{1}{f}\right)
$$

${ }^{3} \mathrm{Si} d=\frac{A}{\sigma_{A}} \cdot\left(1-\frac{\gamma \cdot \frac{D}{A C} \cdot \frac{A C}{A}}{1+\gamma}\right)$, donde AC indica el valor contable del activo, $\mathrm{y}, \sigma=\frac{\sigma_{A}}{A}$ es la desviación típica de la rentabilidad del activo. Tenemos, $\mathrm{I}=\frac{D}{A C}$ y $b=\frac{A}{A C}$. 


\section{Conclusiones}

En este trabajo, se ha analizado a la empresa en un horizonte perpetuo, pudiéndose así valorar la opción de responsabilidad limitada para el caso habitual en que la vida de la empresa no tiene un horizonte finito. Se ha incorporado a la opción de responsabilidad limitada la opción de abandonar y los costes fijos, lo cual permite analizar simultáneamente la quiebra y el cese de actividades de la empresa.

Así pues, se ha analizado diversos aspectos de la relación entre el valor de las acciones y valor de la deuda por medio de la teoría de opciones, utilizando como concepto fundamental para la modelización la opción de responsabilidad limitada de los accionistas e incorporando opciones de barrera a fin de poder formalizar diversos matices que afectan al tratamiento de la deuda empresarial y a situaciones de dificultades financieras y de gestión, tales como el cese de la actividad empresarial, la suspensión de pagos y la quiebra.

En concreto, podemos decir que las aportaciones de este análisis son las siguientes:

La opción de responsabilidad limitada puede ser concebida como una opción de venta americana perpetua y valorada a través de la valoración de Merton(1973).

El valor máximo del activo que justifica el ejercicio anticipado de la opción de responsabilidad limitada en un horizonte perpetuo, constituye el valor del activo para declarar la quiebra de la empresa.

La opción de abandonar se puede asimilar a una opción de venta americana perpetua con un precio de ejercicio igual a la suma de los costes fijos más el valor de liquidación y con un activo subyacente igual a la diferencia entre los ingresos y los costes variables. Esta opción también puede ser valorada a través de la formula de Merton.

La modelización mediante opciones de los efectos sobre el valor de la empresa de la deuda financiera, los costes fijos y el valor de liquidación permite analizar las causas y las consecuencias para accionistas y acreedores del cese de actividades y la quiebra de la empresa, presentándose tres posibles casos: quiebra sin cese, cese sin quiebra y cese y quiebra simultáneos.

De forma contraria al caso del horizonte finito, en el caso de horizonte perpetuo el punto de ejercicio de la quiebra está en función de la desviación típica de la rentabilidad del activo de la empresa. Esto nos permite relacionar el ratio contable de endeudamiento y el ratio de mercado: valor de mercado del activo/ valor contable del activo. A partir de este modelo perpetuo podemos calcular la distancia de la quiebra. 


\section{ApÉNDICE: VALORACIÓN DE LA OPCIÓN DE RESPONSABILIDAD LIMITADA CON OPCIÓN DE ABANDONAR EN EL CASO DE CONSIDERACIÓN EXPLÍCITA DE LOS COSTES FIJOS}

La opción de responsabilidad limitada con opción de abandonar cuando se consideran los costes fijos consiste en una opción de venta cuyo activo subyacente, $A S$, viene dado por la suma del activo con la opción de abandonar que incorpora el efecto de los costes fijos, $A O$. De acuerdo con la ecuación (13), el valor del activo subyacente de esta opción es el siguiente:

$$
A S=A+\frac{L V+S D}{1+\gamma} \cdot\left(\frac{(1+\gamma) \cdot(A+S D)}{\gamma \cdot(L V+S D)}\right)
$$

$A$, el valor de mercado de las inversiones de la empresa, sigue el proceso estocástico de Ito, de acuerdo con la hipótesis generalmente aceptada y asumida en este trabajo. $A S$ es función de $A$. Según el lema de Ito (Hull, 2000 pág. 230), una función de una variable estocástica que sigue un proceso de Ito, sigue también un proceso de Ito.

Nos permite aplicar el lema Ito para determinar el proceso estocástico del activo subyacente $A S$ :

$$
d A S=\left(\frac{\partial A S}{\partial A} \cdot \mu \cdot A+\frac{\partial A S}{\partial t}+\frac{1}{2} \cdot \frac{\partial^{2} A S}{\partial A^{2}} \cdot \sigma^{2} \cdot A^{2}\right) \cdot d t+\cdot d z
$$

donde $\left(\frac{\partial A S}{\partial A} \cdot \mu \cdot A+\frac{\partial A S}{\partial t}+\frac{1}{2} \cdot \frac{\partial^{2} A S}{\partial A^{2}} \cdot \sigma^{2} \cdot A^{2}\right)$ es la tasa de tendencia, $\left(\frac{\partial A S}{\partial A} \cdot \sigma \cdot A\right)^{2}$ es la tasa de varianza y $d z$ sigue un proceso de Wiener.

El cálculo de las derivadas de $A S$, proporciona los siguientes valores:

$$
\frac{\partial A S}{\partial A}=1-\left(\frac{(A+S D) \cdot(1+\gamma)}{(S D+L V) \cdot \gamma}\right)^{-1-\gamma}
$$




$$
\frac{\partial^{2} A S}{\partial A^{2}}=\frac{(S D+L V) \cdot \gamma \cdot\left(\frac{(A+S D) \cdot(1+\gamma)}{(S D+L V) \cdot \gamma}\right)^{-\gamma}}{(A+S D)^{2}}
$$

$\frac{\partial A S}{\partial t}=0$, por tratarse de una opción perpetua

Con lo que el proceso estocástico de $A S$ resulta ser:

$$
\begin{aligned}
& d A S=\left(\left(1-\left(\frac{(A+S D) \cdot(1+\gamma)}{(S D+L V) \cdot \gamma}\right)^{-1-\gamma}\right) \cdot \mu \cdot A+\frac{1}{2} \cdot \frac{(S D+L V) \cdot \gamma \cdot\left(\frac{(A+S D) \cdot(1+\gamma)}{(A+S D) \cdot \gamma}\right)^{-\gamma}}{(A+S D)^{2}} \cdot \sigma^{2} \cdot A^{2}\right) \cdot d t \\
& +\left(1-\left(\frac{(A+S D) \cdot(1+\gamma)}{(S D+L V) \cdot \gamma}\right)^{-1-\gamma}\right) \cdot \sigma \cdot A \cdot d z
\end{aligned}
$$

El proceso estocástico de la rentabilidad de $A S$ es:

$\frac{\partial A S}{A S}=\frac{\left(\left(1-\left(\frac{(A+S D) \cdot(1+\gamma)}{(S D+L V) \cdot \gamma}\right)^{-1-\gamma}\right) \cdot \mu \cdot A+\frac{1}{2} \cdot \frac{(S D+L V) \cdot \gamma \cdot\left(\frac{(A+S D) \cdot(1+\gamma)}{(A+S D) \cdot \gamma}\right)^{-\gamma}}{(A+S D)^{2}} \cdot \sigma^{2} \cdot A^{2}\right) \cdot d}{A+\frac{L V+S D}{1+\gamma} \cdot\left(\frac{(1+\gamma) \cdot(A+S D)}{\gamma \cdot(L V+S D)}\right)} \cdot d t+$ $\frac{\left(1-\left(\frac{(A+S D) \cdot(1+\gamma)}{(S D+L V) \cdot \gamma}\right)^{-1-\gamma}\right) \cdot \sigma \cdot A}{A+\frac{L V+S D}{1+\gamma} \cdot\left(\frac{(1+\gamma) \cdot(A+S D)}{\gamma \cdot(L V+S D)}\right)} \cdot d z$

Realizando las correspondientes operaciones, resulta: 


$$
\begin{aligned}
& \frac{d A S}{A S}=\frac{A \cdot \mu+\frac{A \cdot\left(\frac{(A+S D) \cdot(1+\gamma)}{(S D+L V) \cdot \gamma}\right)^{-1-\gamma} \cdot\left(-2 \cdot(A+S D) \cdot \mu+A \cdot(1+\gamma) \cdot \sigma^{2}\right)}{2 \cdot(A+S D)}}{A+\frac{(S D+L V) \cdot\left(\frac{(A+S D) \cdot(1+\gamma)}{(A+S D) \cdot \gamma}\right)^{-\gamma}}{1+\gamma}} \cdot d t+ \\
& \frac{A \cdot\left(1-\left(\frac{(A+S D) \cdot(1+\gamma)}{(S D+L V) \cdot \gamma}\right)^{-1-\gamma}\right)}{A+\frac{(S D+L V) \cdot\left(\frac{(A+S D) \cdot(1+\gamma)}{(A+S D) \cdot \gamma}\right)^{-\gamma}}{(1+\gamma} \sigma \cdot d z}
\end{aligned}
$$

Por tanto, el valor esperado de la rentabilidad de $A S(\mu$ ') y su desviación típica $\left(\sigma^{\prime}\right)$, son:

$$
\begin{gathered}
\mu^{\prime}=\frac{A \cdot \mu+\frac{A \cdot\left(\frac{(A+S D) \cdot(1+\gamma)}{(S D+L V) \cdot \gamma}\right)^{-1-\gamma} \cdot\left(2 \cdot(A+S D) \cdot \mu+A \cdot(1+\gamma) \cdot \sigma^{2}\right)}{2 \cdot(A+S D)}}{A+\frac{(S D+L V) \cdot\left(\frac{(A+S D) \cdot(1+\gamma)}{(A+S D) \cdot \gamma}\right)^{-\gamma}}{1+\gamma}} \\
\sigma^{\prime}=\frac{A \cdot\left(1-\left(\frac{(A+S D) \cdot(1+\gamma)}{(S D+L V) \cdot \gamma}\right)^{-1-\gamma}\right)}{A+\frac{(S D+L V) \cdot\left(\frac{(A+S D) \cdot(1+\gamma)}{(A+S D) \cdot \gamma}\right)^{-\gamma}}{1+\gamma} \sigma}
\end{gathered}
$$

Que es el resultado de la ecuación (18).

Para calcular el valor de la opción de responsabilidad limitada con opción de abandonar en este caso que consideramos explícitamente los costes fijos, procedemos a sustituir en la fórmula de Merton el valor del activo subyacente, la desviación típica y el precio de ejercicio, obteniendo: 


$$
F_{O A}=\frac{D}{1+\gamma^{\prime}} \cdot\left[\frac{\left(1+\gamma^{\prime}\right) \cdot A S}{\gamma^{\prime} \cdot D}\right]^{-\gamma^{\prime}}
$$

donde:

$$
\gamma^{\prime}=\frac{2 \cdot r}{\sigma^{\prime 2}}
$$

Sustituyendo $A S$ por su valor de acuerdo a (13), resulta:

$$
F_{O A}=\frac{D}{1+\gamma^{\prime}} \cdot\left[\frac{\left(1+\gamma^{\prime}\right) \cdot\left(A+\frac{L V+S D}{1+\gamma} \cdot\left(\frac{(1+\gamma) \cdot(A+S D)}{\gamma \cdot(L V+S D)}\right)\right)}{\gamma^{\prime} \cdot D}\right]^{-\gamma^{\prime}}
$$

Es el valor de la ecuación (23) del texto.

\section{REFERENCIAS BIBLIOGRÁFICAS:}

BJork, Tomas (1998), Arbitrage Theory in Continuous Time, Oxford, Oxford University Press.

BLACK, Fisher y Cox, John C. (1976), "Valuing corporate securities: some effects on bond indenture provisions», The Journal of Finance, vol. 31, núm. 2, 351-367.

Black, Fisher y Scholes, Myron (1973), «The princing of options and corporate liabilities», Journal of Political Economy, 81, 637-654.

Benson, R. y Daniel, N. (1991), «Up, over and out», Risk, junio.

Boyle, P. P. y Lau, S. H. (1994), «Bumping up against the barrier with the binomial method», Journal of Derivatives, vol. 1, núm. 4, 6-14.

Brennan, Michael y Schwartz, Eduard S. (1978), «Corporate income taxes, valuation, and the problem of optimal capital structure», Journal of Business, vol. 51, núm. 1, 103-114.

Brealey, Richard A. y Myers, C. S. (2000), Principles of Corporate Finance, 8. a edición, McGraw Hill, Nueva York.

Cox, John C. y Rubinstein, Mark (1985), Options Markets, Prentice Hall, Englewood Cliffs, Nueva Jersey.

Crosbie, Peter J. y Bohn, Jeffrey R. (2002), Modelling Default Risk. KMV, www.kmv.com.

Crouny, Michel; Galai, Dan y Mark, Robert (2001), Risk Management, McGraw-Hill, New York.

Dahlstrom, Tom y Mella-Barral, Pierre (2003), «Corporate walkout decisions and the value of default», vol. 7, núm. 3, 325-360. 
Dixit, Avinash K. y PINDYcK, Robert S. (1994), Investment Under Uncertainty, Princenton, New Jersey.

Duffee, Gregory R. (1999), «Estimating the price of default risk», The Review of Financial Studies, vol. 12, núm. 1, 197-226.

Gemmill, Gordon (1993), Options Pricing. An International Perspective, McGraw-Hill, Londres.

Eliasson, A.; GaO, S. y Song, J. (2002), Exercising real options: The case of voluntary liquidations, Documento de trabajo.

Hudson, M. (1991), «The value of going out», Risk, marzo, 29-33.

Hull, John C. (2000), Options, Futures \& Other Derivatives, 4. ${ }^{a}$ edición, Prentice-Hall, Upper Saddle River, New Jersey.

Hull, John C. y White, Alan (1995), «The impact of default risk on the prices of options and other derivative securities», Journal of Banking and Finance, vol. 19, núm. 2, mayo, 299322.

- (2001), «The Estimation of Default Probabilities», en Carol Alexander (ed.), Mastering Risk, Financial Times-Prentice Hall, vol. 2, Londres, págs. 171-18,.

Jones, Philip E.; Mason, Scott P. y Rosenfeld, Eric (1984), «Contingent claims analysis of corporate capital structures: an empirical investigation», The Journal of Finance, vol. 39, núm. 3, 611-627.

LELAND, Hayne E. (1994), «Corporate debt value, bond covenants, and optimal capital structure», The Journal of Finance, vol. 49, núm. 4, 1.213-1.252.

LinTNER, John (1965), «The valuation of risk assets and the selection of risky investments in stock portfolios and capital budgets», Review of Economics and Statistics, vol. 47, núm. 1, febrero, 13-37.

Longstaff, Francis A. y Schwartz, Eduard S. (1995), «A simple approach to valuing risky fixed and floating rate debt», The Journal of Finance, vol. 50, núm. 3, 789-819.

Mella-Barral, Pierre (1999), «The dynamics of default and debt reorganization», Review of Financial Studies, vol. 12, núm. 3, 535-578.

Mella-Barral, Pierre y William Perraudin (1997), «Straategic debt service», The Journal of Finance, vol. 52, $n^{\circ}$ 2, 531-556.

Merton, Robert C. (1973), «The Theory of Rational Option Pricing», Bell Journal of Economics and Management Science, 4, primavera, 141-183.

- (1974), «On the Pricing of Corporate Debt: the Risk Structure of Interest Rates», Journal of Finance, vol. 29, mayo, 449-470.

- (1977), «On the pricing of contingent claims and the Modigliani-Miller theorem», Journal of Financial Economics, vol. 5, noviembre, 241-249.

- (1990), Continuos-Time Finance, 1. a edición revisada, Basil Blackwell, Oxford.

Modigliani, Franco y Millar, Merton H. (1958), «The cost of capital, corporation finance and the theory of investment», American Economic Review, vol. 48, junio, 261-297.

Mossin, Jan (1966), «Equilibrium in a capital asset market», Econometrica, vol. 34, núm. 4, octubre, $768-783$. 
Neftci, S. (1996), Introduction to Mathematics of Financial Derivatives, Academic Press, Nueva York.

Nelken, I. (1996), The Handbook of exotic Options, Irwin, Chicago, Illionis.

PEÑA, Juan Ignacio (2002), La gestión de riesgos financieros de mercado y crédito, PrenticeHall, Madrid.

Rich, Don C. (1994), «The Mathematical Foundations of Barrier Options-Pricing Theory», Advances in Futures and Options Research, vol. 7, 267-311.

RichKen, P. (1995), «On princing barrier options», Journal of Derivatives, vol. 3, núm. 2, invierno, 19-28.

Ross, Stephen A. (1976), «The arbitrage theory of capital asset pricing», Journal of Economic Theory, 13, diciembre, 341-360.

Rubinstein, Mark y Reiner, E. (1991),» Breaking down the barrier», Risk, septiembre, 28-35.

SAunders, Anthony (1999), Credit Risk Measurement, John Wiley\&Sons, New York.

Sharpe, William F. (1964), «Capital asset prices: A theory of market equilibrium under conditions of risk», Journal of Finance, vol. 19, núm. 3, septiembre, 425-442.

Sharpe, William F.; Alexander, Gordon y Bailey, Jeffrey V. (1999), Investments, 6. ${ }^{a}$ edición, Prentice Hall, Upper Saddle River, Nueva jersey.

SNYDER, G.L. (1969), «Alternative forms of options», Financial Analysts Journal, septiembreoctubre.

The Options Institute (ed.) (1999), Options, 3. a edición, McGraw-Hill, Nueva York.

TREYNOR, Jack (1961), «Toward a theory of the market value of risky assets», mimeografía.

WonG, Kit Pong (2006), «The effects of abandonment options on operating leverage and forward hedging», International Review of Economics of Finance, vol. 15, núm. 1, 72-86.

Zhang, P. G. (1997), Exotic Options, Worl Scientific Publishing Co, Londres. 